\title{
Dural Arteriovenous Fistula of the Anterior Cranial Fossa With Carotid Artery Stenosis Treated by Simultaneous Transarterial Embolization and Carotid Artery Stenting
}

\author{
-Case Report-
}

\author{
Hideaki ISHIHARA, Shoichiro ISHIHARA, Hiroaki NEKI, Mai OKAWARA, \\ Ryuzaburo KANAZAWA, Shinya KOHYAMA, and Fumitaka YAMANE \\ Division of Endovascular Neurosurgery, Stroke Center, International Medical Center, \\ Saitama Medical University, Hidaka, Saitama
}

\begin{abstract}
A 71-year-old male presented with a rare case of dural arteriovenous fistula (AVF) of the anterior cranial fossa associated with carotid artery stenosis manifesting as a transient visual disorder. The therapeutic strategy was complicated because the collateral network to the ischemic brain from the external carotid artery was associated with the dural AVF. Transarterial embolization of the shunt was performed simultaneously with carotid artery stent placement. The patient showed good recovery despite a tiny skin ulcer around his nose.
\end{abstract}

Key words: anterior cranial fossa, carotid artery stenosis, dural arteriovenous fistula, transarterial embolization

\section{Introduction}

Dural arteriovenous fistula (AVF) is generally treated by endovascular surgery. However, dural AVF of the anterior cranial fossa should be treated through craniotomy because of the risk associated with embolization of the ophthalmic artery and the inadequate success rate of endovascular surgery. Dural AVF of the anterior cranial fossa is rare, accounting for only $5.8 \%$ of all cases, but with a high bleeding rate of $60-80 \%{ }^{6}$ ) We treated a rare case of dural AVF of the anterior cranial fossa associated with carotid artery stenosis, which required simultaneous intervention by endovascular surgery.

\section{Case Report}

A 71-year-old male complained of transient visual disorder. He was referred to our hospital under a diagnosis of carotid artery stenosis based on the findings of magnetic resonance (MR) imaging. Angiography revealed severe stenosis of the left internal carotid artery (Fig. 1A). The distal flow of the internal carotid artery was supplied by retrograde flow of the left ophthalmic artery. In addition, angiography showed a dural AVF of the anterior cranial fossa mainly fed by the left facial artery and drained into the cortical vein toward the superior sagittal sinus (Fig. 1B, C). The dural AVF also involved many feeders (Fig. 1D).

Received January 7, 2009;

Accepted April 8, 2010
We predicted that circulatory reconstructive surgery of the internal carotid artery would increase flow into the ophthalmic artery, resulting in bleeding from the dural AVF, whereas embolization of the dural AVF would occlude both the feeders and the collateral network from the external carotid artery, resulting in reduced flow to the ischemic brain. Therefore, we planned simultaneous treatment of both lesions. Carotid artery stenting would cause loss of the transarterial access route via the external carotid artery for the treatment of the dural AVF, and would also cause difficulty in injecting the glue because of the antegrade flow of the ophthalmic artery. Therefore, we performed embolization of the dural AVF prior to carotid artery stenting.

Three-dimensional digital subtraction angiography (3DDSA) of the bilateral carotid arteries demonstrated the detailed vascular anatomy around the narrow shunt point (Fig. 2). The left facial artery had divided into three branches and the two medial branches were acting as feeders. The catheters were introduced via the facial artery (Fig. 3A) and selective angiography was performed with manual compression of the nasal dorsal artery to prevent infiltration of the glue into the cutaneous vessels and to control blood flow (Fig. 3B). Change in the catheter tip position visualized the route to the left ophthalmic artery (Fig. 3C) and the route to the shunt point (Fig. 3D). The shunt point was completely embolized by injection of $33 \%$ N-butyl-cyanoacrylate (Fig. 4A, B). The left internal carotid artery stenosis was then treated (Fig. 4C). Angiography showed the left ophthalmic artery had reco- 


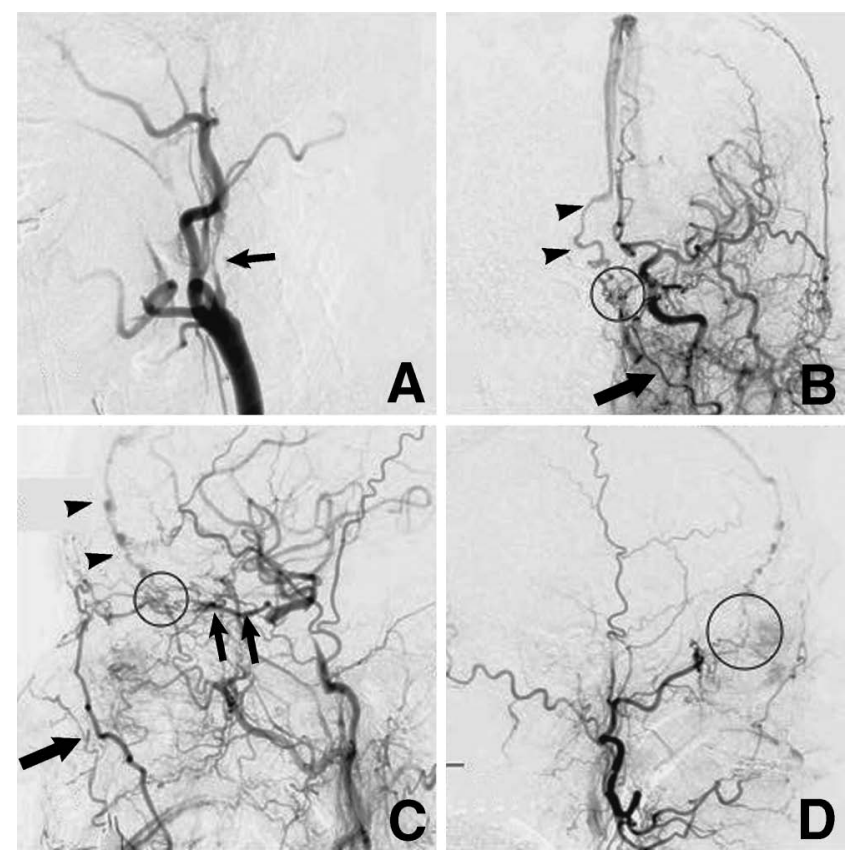

Fig. 1 A: Left internal carotid angiogram showing severe stenosis (arrow). B, C: Left common carotid angiograms, anterior and lateral views, showing a dural arteriovenous fistula (circle) fed mainly by the left facial artery (thick arrow) and draining into the cortical vein (arrowheads) toward the superior sagittal sinus, and retrograde flow of the left ophthalmic artery (arrows) supplying the ischemic brain. D: Right common carotid angiogram, lateral view, showing many feeders concentrated at the shunt point (circle).

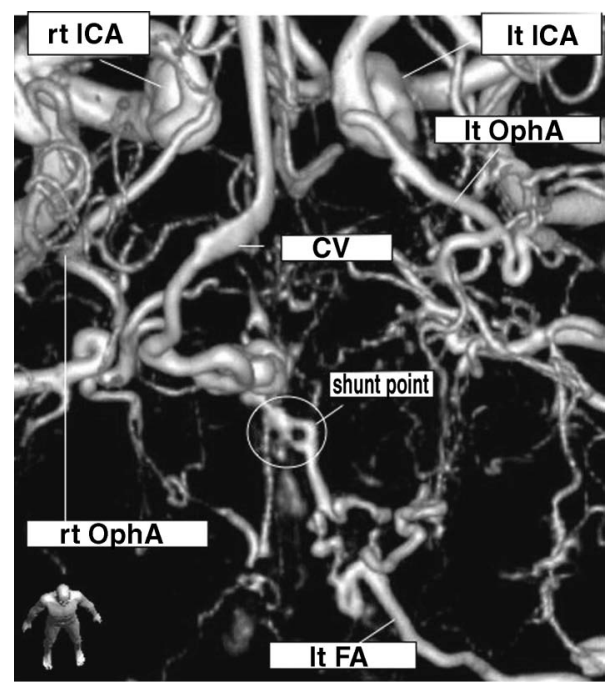

Fig. 2 Three-dimensional digital subtraction angiogram, supero-anterior view, of the bilateral common carotid arteries showing the left facial artery (FA) and the right ophthalmic artery (OphA) mainly flowing to the narrow shunt point (circle) and draining into the cortical vein (CV). The outside peripheral branch of the left FA, which connected with the left OphA, was not involved with the shunt. ICA: internal carotid artery.

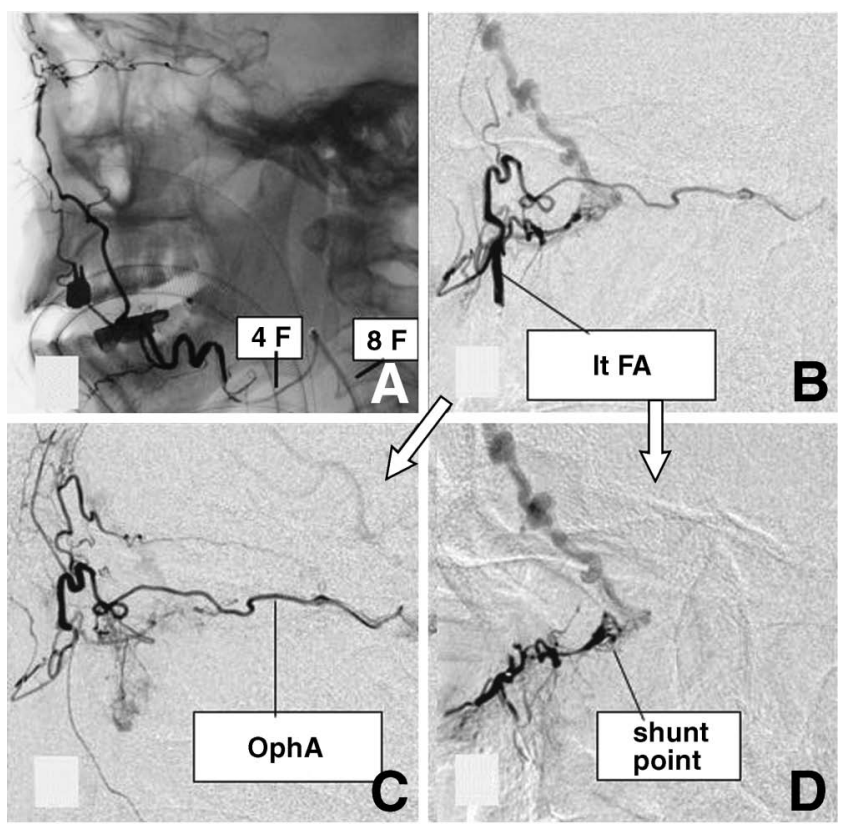

Fig. 3 A: Left facial angiogram showing the transarterial approach via the left facial artery (FA). B-D: Superselective facial artery angiograms, lateral view, with changes in the microcatheter tip position demonstrating various routes to the ophthalmic artery (OphA) and the shunt point (circle).
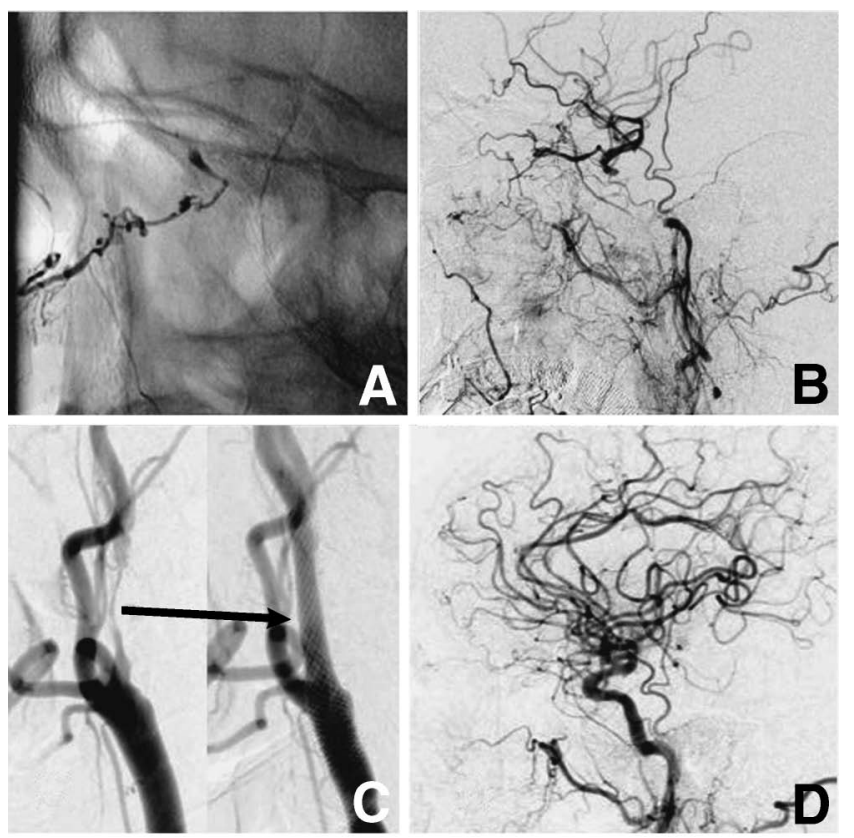

Fig. 4 A: Left facial angiogram showing N-butyl-cyanoacrylate injected into the shunt point and the immediate proximal drainage vein. B: Left common carotid angiogram, lateral view, showing complete occlusion of the dural arteriovenous fistula. C: Left internal carotid angiogram showing stent placement (arrow). D: Postoperative left common carotid angiogram showing the antegrade left ophthalmic artery without shunt flow. 
vered antegrade flow, whereas the shunt was not visualized (Fig. 4D).

The patient had a tiny skin ulcer around the root of his nose, which cleared up rapidly. Postoperative MR imaging did not demonstrate cerebral infarction and the patient was discharged a week later. Cerebral angiography after 3 months could not detect the dural AVF.

\section{Discussion}

Only two previous cases of dural AVF of the anterior cranial fossa have been associated with occlusion ${ }^{4)}$ or moderate stenosis ${ }^{5)}$ of the internal carotid artery, both manifesting as cerebral infarction with the dural AVF treated by delayed craniotomy. In the present case, the feeder of the dural AVF was associated with the collateral network responsible for the ischemia, and simultaneous treatment of both lesions was essential because separate procedures would have caused bleeding from the dural AVF or led to cerebral infarction due to the internal carotid artery stenosis. The collateral network causing the ischemia might have been involved in the development of the dural AVF, but we presumed that the association was incidental.

Dural AVF of the anterior cranial fossa is generally treated by craniotomy, ${ }^{7,9)}$ but this procedure carries some risks of complications, such as olfactory nerve injury, cerebrospinal fluid leakage, and infection. ${ }^{3)}$ Termination of antiplatelet therapy before craniotomy might cause cerebral infarction. Recently, dural AVF of the anterior cranial fossa was treated by embolization with glue. ${ }^{8)}$ Transvenous embolization is possible, but manipulation of the tortuous fragile vein is associated with the risk of vessel rupture. ${ }^{2)}$ Most of the shunt points are narrow around the crista galli, ${ }^{1}$ including that in our case. The retrograde flow of the ophthalmic artery carried the risk of migration of the glue, but was also advantageous in allowing the glue to reach the shunt point. Therefore, we concluded that transarterial embolization was the best approach.

Modern fluoroscopy allows analysis of complicated vascular structures and 3D-DSA easily identified the shunt point despite the complicated vascular structure. Since selective angiography of the main feeder demonstrated the shunt point, we did not need flow control or particle embolization of other feeders. However, we need to pay careful attention to the ophthalmic artery and the dangerous anastomosis, and to the cutaneous vessels during glue injection. Steady manual compression of the vessels around the skin is necessary to prevent infiltration of the glue into the skin.

Dural AVF of the anterior cranial fossa can be treated by transarterial embolization based on accurate visualization of the vascular structure by 3D-DSA.

\section{References}

1) Abrahams JM, Bagley LJ, Flamm ES, Hurst RW, Sinson GP: Alternative management considerations for ethmoidal dural arteriovenous fistulas. Surg Neurol 58: 410-416, 2002

2) Defreyne L, Vanlangenhove P, Vandekerckhove T, Deschrijver I, Sieben G, Klaes R, Kunnen M: Transvenous embolization of a dural arteriovenous fistula of the anterior cranial fossa: preliminary results. AJNR Am J Neuroradiol 21: 761765,2000

3) Deschler DG, Gutin PH, Mamelak AN, McDermott MW, Kaplan MJ: Complications of anterior skull base surgery. Skull Base Surg 6: 113-118, 1996

4) Harada J, Okamoto S, Hamada H, Kubo M, Kuwayama N, Endo S: [Dural arteriovenous fistula of the anterior cranial fossa associated with occlusion of the internal carotid artery: a case report]. No Shinkei Geka 29: 421-425, 2001 (Japanese)

5) Kawaguchi T, Kawano T, Honnma T, Kaneko Y, Tsutsumi M, Dousaka A, Ooigawa H, Kazekawa K: [Non-hemorrhagic dural arteriovenous malformations of the anterior cranial fossa]. Surgery for Cerebral Stroke 27: 24-30, 1999 (Japanese)

6) King WA, Martin NA: Intracerebral hemorrhage due to dural arteriovenous malformations and fistulae. Neurosurg Clin N Am 3: 577-590, 1992

7) Lawton MT, Chun J, Wilson CB, Halbach VV: Ethmoidal dural arteriovenous fistulae: an assessment of surgical and endovascular management. Neurosurgery 45: 805-811, 1999

8) Lv X, Li Y, Wu Z: Endovascular treatment of anterior cranial fossa dural arteriovenous fistula. Neuroradiology 50: 433-437, 2008

9) Shinohara Y, Yoshimoto T, Fukuuchi Y, Ishigami S: [Japanese Guidelines for the Management of Stroke]. Tokyo, Kyowakikaku, 2004, pp 123-124 (Japanese)

Address reprint requests to: Hideaki Ishihara, M.D., Division of Endovascular Neurosurgery, Stroke Center, International Medical Center, Saitama Medical University, 1397-1 Yamane, Hidaka, Saitama 350-1298, Japan.

e-mail: hideaki@saitama-med.ac.jp 